\title{
Clinical Lipid Control Success Rate Before and After Percutaneous Coronary Intervention in Iran; a Single Center Study
}

\author{
Seyed Kianoosh Hosseini ${ }^{1}$, Maryam Tahvildari ${ }^{2}$, Mohammad Javad Alemzadeh Ansari ${ }^{1}$, \\ Manouchehr Nakhjavani ${ }^{3,{ }^{*}}$, Alireza Esteghamati ${ }^{3}$, Masoumeh Lotfi Tokaldany ${ }^{4}$ \\ ${ }_{2}^{1}$ Department of Cardiology, Tehran Heart Center, Tehran University of Medical Sciences, Tehran, IR Iran. \\ 2 Tehran University of Medical Sciences, Tehran, IR Iran. \\ 3 Endocrinology and Metabolism Research Centre (EMRC), Vali-Asr Hospital, Tehran University of Medical Sciences, Tehran, IR Iran. \\ 4 Research Department, Tehran Heart Center, Tehran University of Medical Sciences, Tehran, IR Iran \\ ${ }^{*}$ Corresponding author: Manouchehr Nakhjavani, Endocrinology and Metabolism Research Centre (EMRC), Vali-Asr Hospital, Tehran University of Medical Sciences, Tehran, IR Iran. \\ Tel:+98-9121098214, E-mail: nakhjavanim@tums.ac.ir.
}

Received: May 06, 2012; Revised: April 12, 2013; Accepted: April 25, 2013

\begin{abstract}
Background: High cholesterol levels have long been considered an independent risk factor for cardiovascular disease(CVD). Objective: Controlling risk factors such as dyslipidemia in patients with coronary artery disease is necessary. We aimed to evaluate the success rate of lipid control, during 9 months follow-up after percutaneous coronary intervention (PCI).

Patients and Methods: A total of 195 patients (67.7\% men, mean age $=57.8 \pm 9.4$ years) who underwent $\mathrm{PCI}$ in Tehran Heart Center were included. Serum lipid profiles were measured in all the patients before PCI and at 9-month follow-up. Dyslipidemia was defined as serum levels of LDL-C $\geq 100$ or TG $\geq 150$ or TC $\geq 200$ or HDL-C $\leq 40 \mathrm{mg} / \mathrm{dl}$ in the men and $\leq 50 \mathrm{mg} / \mathrm{dl}$ or less in the women, or non-HDL-C $\geq$ $130 \mathrm{mg} / \mathrm{dl}$ with or without the consumption of lipid-lowering agents. During follow up, all patients were given atorvastatin 20-40 $\mathrm{mg} / \mathrm{day}$. Results: Overall, $26.2 \%$ had diabetes mellitus, $42.6 \%$ had hypertension, and 34.9\% were smokers. Dyslipidemia was more common in the women. At 9-month follow-up, there was no significant changes in terms of the prevalence of high HDL-C or low TG in patients; however, a significant increase was seen in the prevalence low TC in patients (63.6\%vs. $80.5 \%$; p value $<0.001)$, LDL-C (47.2\% vs. $65.6 \%$; p value $<0.001$ ), and non-HDL-C (40.0\% vs. 63.1\%; p value $<0.001)$.

Conclusions: Although by current treatments, the prevalence of patients with low TC, LDL-C and non-HDL-C has significantly increased; dyslipidemia persisted in a considerable proportion of patients. These results necessitate further investigations into the relationship between high serum lipids and long-term outcome of patients after PCI as well as further evaluations of the dyslipidemia treatment strategies.
\end{abstract}

Keywords: Dyslipidemia; Lipid Control; Percutaneous Coronary Intervention

\section{Background}

High cholesterol levels have long been considered an independent risk factor for cardiovascular disease (CVD) (1). It has been demonstrated that high total cholesterol (TC), high triglyceride (TG), high low-density lipoprotein cholesterol (LDL-C), low high-density lipoprotein cholesterol (HDL-C) and high non-HDL-C levels are associated with a high incidence rate of CVD (1-5). Patients undergoing percutaneous coronary intervention (PCI) are amongst the individuals with highest risk for developing adverse cardiac events and higher morbidity and mortality. Therefore, meticulous follow-up sessions and patient education are said to be crucial in further reaching the target levels of blood lipids in this high-risk group of patients. In spite of the emphasis of guidelines on the tight con- trol of the aforementioned modifiable risk factors, several surveys have shown that still a large number of patients have not achieved their optimal status (6-9). There are different reports in various studies about the rates of dyslipidemia control (6, 8-11). The aim of this study was to evaluate the lipid profile levels (LDL-C, HDL-C, non-HDL-C, TC, and TG) amongst patients before PCI and during follow-up to estimate our success rates in the control of dyslipidemia. Strength of our study is our Iran population sample, which allows comparison between reportedly encountered data of developed countries and a different ethnicity of a developing country.

\section{Objectives}

Controlling risk factors such as dyslipidemia in patients

Implication for health policy/practice/research/medical education:

Patients undergoing percutaneous coronary intervention (PCI) are amongst the individuals with highest risk for developing adverse cardiac events and higher morbidity and mortality. There are different reports in various studies about the rates of dyslipidemia control. Present study concluded that lipidlowering agents and regular follow-up visits did assist in achieving lipid-control goals in our patients, but a large proportion of the patients remained dyslipidemic at the end of the follow-up period. More attention should, therefore, be paid to life style modification, using statins with higher doses and utilizing other classes of lipid-lowering agents such as ezetimibe or fibrates, if needed, in order to achieve the optimal targets of LDL-C, HDL-C, and TG.

Copyright (C) 2013, Iranian Red Crescent Medical Journal; Licensee Kowsar Ltd. This is an Open Access article distributed under the terms of the Creative Commons Attribution License (http://creativecommons.org/licenses/by/3.0), which permits unrestricted use, distribution, and reproduction in any medium, provided the original work is properly cited. 
with coronary artery disease is necessary. We aimed to evaluate the success rate of lipid control, during 9-month follow-up after percutaneous coronary intervention (PCI).

\section{Patients and Methods}

This retrospective study was conducted on patients who were referred by cardiologists to Tehran Heart Center both from inside and outside of the center and underwent PCI between 2003 and 2007. All the medical records of the patients were derived from the Interventional Registry carried out at Tehran Heart Center (THCR-IC). The databank contains patient data collected by cardiologists and trained general practitioners, and the validity of all the data is checked by re abstracting $10 \%$ of the patients entries and by reentering $5 \%$ of the patients' records. During this period, 4,732 patients underwent PCI, but full data on the lipid profile including before and after PCI were available in only 195 patients. Demographic characteristics and laboratory data were gathered at the time of the administration. This research was approved by the institutional review board, overseeing the participation of human subjects in research at Tehran University of Medical Sciences. This study conformed to the principles outlined in the Declaration of Helsinki. Lipid-lowering agent, atorvastatin 20-40 mg per day was commenced at the time of admission, if not given before hospitalization, and they were continued after PCI and discharge from hospital. All the patients were invited to return to our follow-up clinic. Follow-up information was obtained through direct clinical visits of the patients for 9 months after the procedures or from the referring physicians and telephone interviews. Dyslipidemia was positive if at least one of these conditions existed: TC of $200 \mathrm{mg} / \mathrm{dl}$ or more, LDL-C of $100 \mathrm{mg} / \mathrm{dl}$ or more, TG of $150 \mathrm{mg} / \mathrm{dl}$ or more, HDL-C of $40 \mathrm{mg} / \mathrm{dl}$ or less in men and $50 \mathrm{mg} / \mathrm{dl}$ or less in women, and non-HDL-C of $130 \mathrm{mg} / \mathrm{dl}$ or more. Achieving success in lipid control was defined as reaching the target levels for each of the above-mentioned components of the lipid profile. The lipid control success rates were evaluated before PCI and at 9-month follow-up.

\subsection{Statistical Methods}

The data are presented as mean \pm SD for the continuous variables and as frequencies (percentage) for the categorical variables. The statistical package for social sciences (SPSS) version 15 software was used for data analysis. The continuous variables were compared using the Student t-test and the categorical variables were compared using the Chi-Square or Fisher exact test. The significant differences between the groups were determined at level $<$ 0.05 .

\section{Results}

Amongst the 195 patients, who underwent PCI, 132 (67.7\%) were men. The baseline demographic characteristics of patients before PCI are listed in Table1. Except for smoking, all the other cardiovascular risk factors, including obesity, hypertension, diabetes mellitus, and dyslipidemia, were significantly more frequent in women than in men. In addition, the serum levels of TC, LDL-C, HDL-C and non-HDL-C were significantly higher in women. High TC, TG, LDL-C, non-HDL-C, and low HDL-C were seen in $36.4 \%, 56.9 \%, 52.8 \%, 60.0 \%$, and $47.7 \%$ of the individuals, respectively. Figure 1 shows the prevalence of dyslipidemia before and after PCI. More women were dyslipidemic with respect to LDL-C, HDL-C, and non-HDL-C before and after PCI. The prevalence of patients with high TC and TG was higher in women than that in the men before PCI, but this difference disappeared after the follow-up (Figure 1).

\begin{tabular}{|c|c|c|c|c|}
\hline Characteristics & Total & $\operatorname{Men}(132,67.7 \%)$ & Women $(63,32.3 \%)$ & Pvalue \\
\hline Age, $y$, Mean \pm SD & $57.8 \pm 9.4$ & $57.6 \pm 9.8$ & $58.4 \pm 8.7$ & 0.52 \\
\hline BMI, Kg/m2, Mean \pm SD & $27.5 \pm 4.1$ & $26.7 \pm 3.4$ & $29.4 \pm 4.7$ & $<0.001$ \\
\hline Hypertension, No. (\%) & $83(42.6 \%)$ & $43(32.6 \%)$ & $40(63.5 \%)$ & $<0.001$ \\
\hline Diabetes Mellitus, No.(\%) & $51(26.2 \%)$ & $23(17.4 \%)$ & $28(44.4 \%)$ & $<0.001$ \\
\hline Smoking, No. (\%) & $68(34.9 \%)$ & $64(48.5 \%)$ & $4(6.4 \%)$ & $<0.001$ \\
\hline $\begin{array}{l}\text { Family History for Prema- } \\
\text { ture CVD, No. (\%) }\end{array}$ & $54(27.7 \%)$ & $33(25 \%)$ & $21(33.3 \%)$ & 0.22 \\
\hline FBS, g/dl, Mean \pm SD & $117.5 \pm 48.7$ & $111.0 \pm 42.8$ & $131.4 \pm 57.4$ & 0.008 \\
\hline Hb, g/dl, Mean \pm SD & $13.5 \pm 1.7$ & $14.1 \pm 1.4$ & $12.1 \pm 1.4$ & $<0.001$ \\
\hline \multicolumn{5}{|l|}{ Lipid Profile, Mean \pm SD } \\
\hline $\mathrm{TC}^{\mathrm{a}}, \mathrm{mg} / \mathrm{dl}$ & $185.9 \pm 49.5$ & $179.2 \pm 51.6$ & $199.9 \pm 41.8$ & 0.006 \\
\hline HDL-C ${ }^{\mathrm{a}}, \mathrm{mg} / \mathrm{dl}$ & $42.6 \pm 9.4$ & $41.4 \pm 9.2$ & $45.0 \pm 9.6$ & 0.01 \\
\hline LDL-C ${ }^{\mathrm{a}}, \mathbf{m g} / \mathbf{d l}$ & $107.3 \pm 42.3$ & $102.3 \pm 44.6$ & $117.7 \pm 35.2$ & 0.01 \\
\hline $\mathrm{TG}^{\mathrm{a}}, \mathrm{mg} / \mathrm{dl}$ & $175.5 \pm 75.0$ & $169.4 \pm 72.2$ & $188.3 \pm 79.7$ & 0.10 \\
\hline Non-HDL-C ${ }^{\mathrm{a}}, \mathrm{mg} / \mathrm{dl}$ & $143.3 \pm 46.9$ & $137.8 \pm 49.5$ & $154.6 \pm 38.8$ & 0.01 \\
\hline
\end{tabular}

\footnotetext{
a Abbreviations: TC, total cholesterol; HDL-C, high-density lipoprotein cholesterol; LDL-C, low-density lipoprotein cholesterol; TG, triglyceride; nonHDL-C, non high-density lipoprotein cholesterol
} 


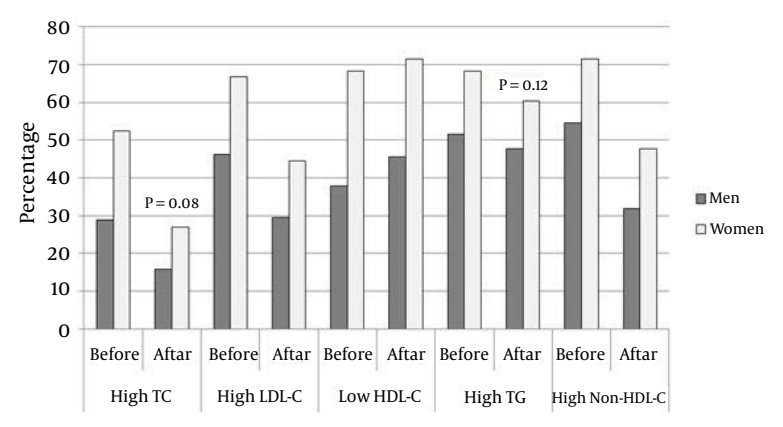

Figure 1. Comparison Between men and Women in Terms of the Prevalence of Dyslipidemia

Before and after PCI: More women dyslipidemic regarding LDL-C, HDL-C, and non-HDL-C before and after PCI. Although high TC and TG rates were more frequent in women than those in men before PCI, this difference disappeared after the follow-up period. After PCI and discharge from hospital, all the patients went through regular follow-up for 9 months. In this period, the serum concentration of TC, LDL-C and non-HDL-C decreased significantly (185.9 $\pm 49.5 \mathrm{mg} / \mathrm{dl}$ vs. $163.6 \pm 40.9 \mathrm{mg} / \mathrm{dl}$; P value < 0.001), $(107.3 \pm 42.3 \mathrm{mg} / \mathrm{dl}$ vs. $90.3 \pm 41.4 \mathrm{mg} / \mathrm{dl}$; $\mathrm{P}$ value $<0.001)$, and $(143.3 \pm 46.9 \mathrm{mg} / \mathrm{dl}$ vs. $123.5 \pm 48.2 \mathrm{mg} /$ $\mathrm{dl}$; P value $<0.001)$ respectively, whereas there was no significant change in the concentration of HDL-C (42.6 \pm 9.4 $\mathrm{mg} / \mathrm{dl}$ vs. $42.0 \pm 9.0 ; \mathrm{P}$ value $=0.48)$ and $\mathrm{TG}(175.5 \pm 75.0 \mathrm{mg} /$ dl vs. 166.7 \pm 78.9 ; P value $=0.12$ ) (Figure 2 ).

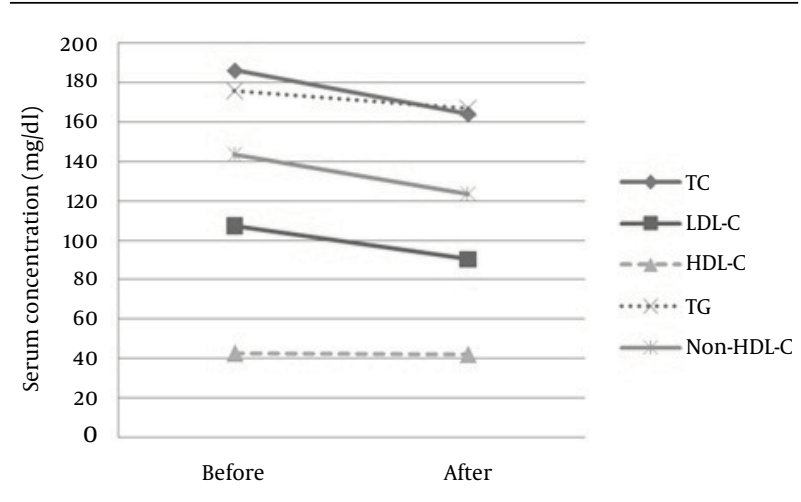

Figure 2. Serum Concentration of Lipids, Before and After PCI

Although the serum concentration of TC, LDL-C and non-HDL-C decreased significantly after 9 months, there was no significant change in the concentration of HDLC and TG. At the time of hospitalization, only 25 (12.8\%) patients (22 men and 3 women; P value $=0.02$ ) had all the measured serum lipids within the acceptable range, whilst after the follow-up period, this figure rose to 40 $(20.5 \%)$ patients ( 32 men and 8 women; $P$ value $=0.08$ ). In contrast, $21(10.8 \%$ ) patients ( 7 men and 14 women; P value $=0.0006$ ) had all the measured serum lipids within the uncontrolled range at the time of hospitalization, and $17(8.7 \%)$ patients ( 8 men and 9 women; $P$ value $=0.06$ ) still remained dyslipidemic after the follow-up. Table 2 depicts the lipid control success rates before PCI and after the follow-up. A significant increase was observed in the prevalence of the patients who had low TC, LDL-C, and non-HDL-C after the follow-up, whereas no significant difference was found in the prevalence of the patients who had high HDL-C and low TG.

\begin{tabular}{|c|c|c|c|c|c|}
\hline Lipid Profile & Values & Sex & Before PCI, No. (\%) & After PCI, No. (\%) & Pvalue \\
\hline \multirow[t]{3}{*}{$\mathbf{T C}^{\mathrm{a}}$} & $<200 \mathrm{mg} / \mathrm{dl}$ & Men & $94(71.2 \%)$ & $111(84.1 \%)$ & 0.01 \\
\hline & & Women & $30(47.6 \%)$ & $46(73.0 \%)$ & 0.006 \\
\hline & & Total & $124(63.6 \%)$ & $157(80.5 \%)$ & $<0.001$ \\
\hline \multirow[t]{3}{*}{ LDL-C $C^{\mathrm{a}}$} & $<100 \mathrm{mg} / \mathrm{dl}$ & Men & $71(53.8 \%)$ & $93(70.5 \%)$ & 0.007 \\
\hline & & Women & $21(33.3 \%)$ & $35(55.6 \%)$ & 0.01 \\
\hline & & Total & $92(47.2 \%)$ & $128(65.6 \%)$ & $<0.001$ \\
\hline \multirow[t]{3}{*}{ HDL-C ${ }^{a}$} & $>40 \mathrm{mg} / \mathrm{dl}$ in men, $>50 \mathrm{mg} / \mathrm{dl}$ in women & Men & $82(62.1 \%)$ & $72(54.5 \%)$ & 0.26 \\
\hline & & Women & $20(31.7 \%)$ & $18(28.6 \%)$ & 0.84 \\
\hline & & Total & $102(52.3 \%)$ & $90(46.2 \%)$ & 0.27 \\
\hline \multirow[t]{3}{*}{$\mathbf{T G}^{\mathrm{a}}$} & $<150 \mathrm{mg} / \mathrm{dl}$ & Men & $64(48.5 \%)$ & $69(52.3 \%)$ & 0.62 \\
\hline & & Women & $20(31.7 \%)$ & 25 (39.7\%) & 0.45 \\
\hline & & Total & $84(43.1 \%)$ & $94(48.2 \%)$ & 0.36 \\
\hline \multirow[t]{3}{*}{ Non-HDL-C ${ }^{a}$} & $<130 \mathrm{mg} / \mathrm{dl}$ & Men & $60(45.5 \%)$ & $90(68.2 \%)$ & $<0.001$ \\
\hline & & Women & $18(28.6 \%)$ & $33(52.4 \%)$ & 0.01 \\
\hline & & Total & $78(40.0 \%)$ & $123(63.1 \%)$ & $<0.001$ \\
\hline
\end{tabular}

a Abbreviations: TC, total cholesterol; HDL-C, high-density lipoprotein cholesterol; LDL-C, low-density lipoprotein cholesterol; TG, triglyceride; nonHDL-C, non high-density lipoprotein cholesterol 


\section{Discussion}

Strong interdependence between high TC, high TG, high LDL-C, low HDL-C, and high non-HDL-C concentrations and CVD has been reported in previous studies $(1-5,12,13)$. On the other hand, the prevalence of cardiovascular risk factors (particularly dyslipidemia) is high amongst the Iranian general population. Azizi et al. reported that the prevalence of high TC, low HDL-C and high TG, amongst the healthy Iranian population was $19.3 \%, 32 \%$, and 5.3\%, respectively (14). In 2008 Ghandehari et al. reported that the prevalence of high LDL-C, high TG, and low HDL-C amongst adult US persons was $28 \%, 13 \%$, and $26 \%$ respectively (15). Ford et al. reported that the prevalence of high TG in the general population 20 years of age or older in the USA was 33.1\% (16). Esteghamati et al. in 2006 showed that the prevalence of high TC, high LDL-C, low HDL-C, and high TG amongst Iranian patients with acute coronary syndrome was $41.8 \%, 63.8 \%, 74.4 \%$, and $49 \%$, respectively (17). Approximately a large proportion of our coronary artery disease patients, who underwent PCI, had high TC (36.4\%), TG (56.9\%), LDL-C (52.8\%), and non-HDL-C (60.0\%), as well as low HDL-C (47.7\%), which chimes in with other studies $(5,17,18)$. Pres et al. in 2010 stated that $60 \%$ of all their patients (diabetic and non-diabetic) with ST-elevation myocardial infarction treated with PCI had high TC; in addition, the prevalence of high TC in the diabetic patients with high LDL-C reached $78 \%$ and in the non-diabetic patients with high LDL-C amounted to $74 \%$. The authors also reported that in their diabetic patients, in-hospital mortality was higher amongst those with elevated LDL$C$ levels and each increase in LDL-C on admission by 1 $\mathrm{mmol} / \mathrm{L}(38.67 \mathrm{mg} / \mathrm{dl})$ was related to an increase in inhospital mortality (5). In our study, by current treatments and after 9 months of follow-up, a 16.9\% increase was seen in the proportion of patients with low TC, $18.4 \%$ increase in the proportion of patients with low LDL-C, and $23.1 \%$ increase in the proportion of patients with low non-HDL-C, whereas there was a drop in the proportion of patients with high HDL-C and there was no significant increase in the proportion of patients with low TG after treatment. Khashayar et al. in 2010 showed that statin usage, albeit capable of reducing TC and LDL levels, did not exert an impact on TG and HDL levels, which is concordant with our results (19). Although after 9 months of follow-up, the proportion of patients with low TC, LDL-C, and nonHDL-C significantly increased in our study, despite using statins in all the patients, 19.5\% had high TC, 34.4\% high LDL-C, 53.8\% low HDL-C, 51.8\% high TG, and 36.9\% high nonHDL-C. Furthermore, in a large proportion of our patients (79.5\%), at least one of the components of the lipid profile remained uncontrollable; this is similar to or even more than that reported previously by other studies (6, 8-10). Stacy et al. in 2006 stated that approximately one third of their patients treated with lipid-lowering agents did not achieve individual lipid goals and two thirds of their patients did not attain the goal for all the three targets (LDL-C, HDL-C, and TG) (8). Goff et al., studying the prevalence, treatment and control of dyslipidemia in people free of clinical CVD, demonstrated that $29.3 \%$ of them had dyslipidemia and only $54.0 \%$ of them reported lipid-lowering drug therapy. Additionally, they showed that the control rate of dyslipidemia was achieved in $75.2 \%$ of the participants with treated dyslipidemia but in only $40.6 \%$ of all the participants with dyslipidemia (10). High prevalence of dyslipidemic patients after PCI in our study may be due to patients poor compliance or statin resistance (20). Amongst our 195 patients with established coronary artery disease, the cardiovascular risk factors, including high body mass index, hypertension, diabetes mellitus, and dyslipidemia, were more common in women; these findings were compatible with those reported by previous studies conducted in Iran $(14,17)$. There are varied concepts about gender-related dyslipidemia. For example, the Cruz et al. study in 2008 reported that the rate of dyslipidemia in patients with ischemic heart disease was equal in both men and women, (21) whereas the Goff et al. study showed that dyslipidemia in people free of known clinical CVD was more common in men than in women and that it was treated and controlled less often in men than in women (10). Our findings are compatible with those of the former study, which also stated that diabetic women had higher TC, LDL-C, TG, non-HDL-C, Lp (a), apo-B, and even HDL-C (22). Also in our study, the prevalence of women with high TC, LDL-C, TG, and non-HDL-C, as well as low HDL-C was significantly more than that in the men before PCI and after the follow-up; the only exceptions were high TC and TG after the follow-up period. The prevalence of men with low TC, LDL, and non-HDL-C increased significantly during the follow-up, which was the same as that in women. Despite treatment with atorvastatin, not only the proportion of the men or women with high HDL-C and low TG did not increase, but even a mild (nonsignificant) reduction (6.1\%) was seen in the prevalence of patients with high HDL-C. Several large trials and metaanalyses have investigated the effects of lipid-lowering statin therapy and have consistently demonstrated that statins are highly effective for lowering LDL-C levels (11, 23-27). Despite achieving target LDL levels in patients treated with statins, they are in risk for the progression of atherosclerosis; this may be due to high TG and/or low HDL-C levels (28-30). In contrast, new findings suggest that although HDL-C levels are useful for initial cardiovascular risk assessment, they are not predictive of residual vascular risk amongst patients treated with potent statin therapy who attain very low LDL-C levels and in addition, there is no benefit from administrating niacin for this group of patients. Retrospective design and small sample size are two limitation of our study. In our study, at 9-month follow-up, the prevalence of patients with low TC, LDL-C and non-HDL-C significantly increased, whereas there was no significant increase in the prevalence of pa- 
tients with high HDL-C or low TG. Lipid-lowering agents and regular follow-up visits did assist in achieving lipidcontrol goals in some patients, but a large proportion of the patients remained dyslipidemic at the end of the follow-up period. More attention should, therefore, be paid to life style modification, using statins with higher doses, and utilizing other classes of lipid-lowering agents such as ezetimibe or fibrates, if needed, in order to achieve the optimal targets of LDL-C, HDL-C, and TG. Further studies with prospective design and larger sample sizes dealing with role of lipid control in long term outcome of PCI is recommended.

\section{Acknowledgements}

There is no acknowledgment.

\section{Authors' Contribution}

Study concept and design: Hosseini, Nakhjavani, Esteghamati. Analysis and interpretation of data: Nakhjavani, Alemzadeh-Ansari, Lotfi Tokaldany. Drafting of the manuscript: Tahvildari, Alemzadeh-Ansari. Critical revision of the manuscript for important intellectual content: Hosseini, Nakhjavani, Alemzadeh-Ansari. Statistical analysis: Alemzadeh-Ansari, Lotfi Tokaldany, Tahvildari.

\section{Financial Disclosure}

There is no financial disclosure.

\section{Funding/Support}

This project was supported by Tehran Heart Center research department, Tehran Heart Center, Tehran University of Medical Sciences, Tehran Iran.

\section{References}

1. Chrysant SG. Stopping the cardiovascular disease continuum: Focus on prevention. World J Cardiol. 2010;2(3):43-9.

2. Arsenault BJ, Rana JS, Stroes ES, Despres JP, Shah PK, Kastelein JJ, et al. Beyond low-density lipoprotein cholesterol: respective contributions of non-high-density lipoprotein cholesterol levels, triglycerides, and the total cholesterol/high-density lipoprotein cholesterol ratio to coronary heart disease risk in apparently healthy men and women. J Am Coll Cardiol. 2009;55(1):35-41.

3. Eyre H, Kahn R, Robertson RM, Clark NG, Doyle C, Hong Y, et al. Preventing cancer, cardiovascular disease, and diabetes: a common agenda for the American Cancer Society, the American Diabetes Association, and the American Heart Association. Circulation. 2004;109(25):3244-55.

4. Grundy SM. Obesity, metabolic syndrome, and cardiovascular disease. J Clin Endocrinol Metab. 2004;89(6):2595-600.

5. Pres D, Gasior M, Lekston A, Gierlotka M, Hawranek M, Tajstra M, et al. Relationship between low-density lipoprotein cholesterol level on admission and in-hospital mortality in patients with STsegment elevation myocardial infarction, with or without diabetes, treated with percutaneous coronary intervention. Kardiol Pol. 2010;68(9):1005-12.

6. Petrella RJ, Merikle E, Jones J. Prevalence and treatment of dyslipidemia in Canadian primary care: a retrospective cohort analysis. Clin Ther. 2007;29(4):742-50.

7. Cooke CE, Hammerash WJ, Jr. Retrospective review of sex differences in the management of dyslipidemia in coronary heart dis- ease: an analysis of patient data from a Maryland-based health maintenance organization. Clin Ther. 2006;28(4):591-9.

8. Stacy TA, Egger A. Results of retrospective chart review to determine improvement in lipid goal attainment in patients treated by high-volume prescribers of lipid-modifying drugs. J Manag Care Pharm. 2006;12(9):745-51.

9. Petrella RJ, Merikle E. A retrospective analysis of the prevalence and treatment of hypertension and dyslipidemia in Southwestern Ontario, Canada. Clin Ther. 2008;30(6):1145-54.

10. Goff DC, Jr, Bertoni AG, Kramer H, Bonds D, Blumenthal RS, Tsai MY, et al. Dyslipidemia prevalence, treatment, and control in the Multi-Ethnic Study of Atherosclerosis (MESA): gender, ethnicity, and coronary artery calcium. Circulation. 2006;113(5):647-56.

11. Wilt TJ, Bloomfield HE, MacDonald R, Nelson D, Rutks I, Ho M, et al. Effectiveness of statin therapy in adults with coronary heart disease. Arch Intern Med. 2004;164(13):1427-36.

12. Grundy SM, Benjamin IJ, Burke GL, Chait A, Eckel RH, Howard BV, et al. Diabetes and cardiovascular disease: a statement for healthcare professionals from the American Heart Association. Circulation.1999;100(10):1134-46.

13. Manninen V, Tenkanen L, Koskinen P, Huttunen JK, Manttari M, Heinonen OP, et al. Joint effects of serum triglyceride and LDL cholesterol and HDL cholesterol concentrations on coronary heart disease risk in the Helsinki Heart Study. Implications for treatment. Circulation.1992;85(1):37-45.

14. Azizi F, Rahmani M, Emami H, Mirmiran P, Hajipour R, Madjid $\mathrm{M}$, et al. Cardiovascular risk factors in an Iranian urban population: Tehran lipid and glucose study (phase 1). Soz Praventivmed. 2002;47(6):408-26.

15. Ghandehari H, Kamal-Bahl S, Wong ND. Prevalence and extent of dyslipidemia and recommended lipid levels in US adults with and without cardiovascular comorbidities: the National Health and Nutrition Examination Survey 2003-2004. Am Heart J. 2008;156(1):112-9.

16. Ford ES, Li C, Zhao G, Pearson WS, Mokdad AH. Hypertriglyceridemia and its pharmacologic treatment among US adults. Arch Intern Med. 2009;169(6):572-8.

17. Esteghamati A, Abbasi M, Nakhjavani M, Yousefizadeh A, Basa AP, Afshar H. Prevalence of diabetes and other cardiovascular risk factors in an Iranian population with acute coronary syndrome. Cardiovasc Diabetol. 2006;5:15.

18. Hosseini SK, Mehrpooya M. Lipid Control before CABG and Its Association with In-Hospital Mortality. Iran Red Crescent Med J. 2011;13(2):106-11.

19. Khashayar P, Mohagheghi A. The correlation between dyslipidemia and coronary artery disease based on angiographic findings in an Iranian population. Acta Med Indones. 2010;42(2):82-5.

20. Krauss RM, Mangravite LM, Smith JD, Medina MW, Wang D, Guo X, et al. Variation in the 3-hydroxyl-3-methylglutaryl coenzyme a reductase gene is associated with racial differences in low-density lipoprotein cholesterol response to simvastatin treatment. Circulation. 2008;117(12):1537-44.

21. Cruz I, Serna C, Real J, Galindo G, Gasco E, Galvan L. Ischemic heart disease and primary care: identifying gender-related differences. An observational study. BMC Fam Pract. 2008;9:60.

22. Nakhjavani M, Esteghamati AR, Heshmat F Esfahanian AR. Dyslipidemia in type 2 diabetes mellitus: More atherogenic lipid profile in women. Acta Medica Iranica. 2006;44(2).

23. Cziraky MJ, Watson KE, Talbert RL. Targeting low HDL-cholesterol to decrease residual cardiovascular risk in the managed care setting. J Manag Care Pharm. 2008;14(8 Suppl):S3-28.

24. Rubins HB, Collins D, Robins SJ. The VA HDL intervention trial: clinical implications. Eur Heart J. 2000;21(14):1113-5.

25. Cannon CP, Braunwald E, McCabe CH, Rader DJ, Rouleau JL, Belder $\mathrm{R}$, et al. Intensive versus moderate lipid lowering with statins after acute coronary syndromes. N Engl J Med. 2004;350(15):1495504.

26. Pedersen TR, Kjekshus J, Berg K, Haghfelt T, Faergeman O, Faergeman G, et al. Randomised trial of cholesterol lowering in 4444 patients with coronary heart disease: the Scandinavian Simvastatin Survival Study (4S). 1994. Atheroscler Suppl. 2004;5(3):81-7.

27. Sever PS, Dahlof B, Poulter NR, Wedel H, Beevers G, Caulfield M, et 
al. Prevention of coronary and stroke events with atorvastatin in hypertensive patients who have average or lower-than-average cholesterol concentrations, in the Anglo-Scandinavian Cardiac Outcomes Trial--Lipid Lowering Arm (ASCOT-LLA): a multicentre randomised controlled trial. Drugs. 2004;64 Suppl 2:43-60.

28. Suadicani P, Hein HO, Gyntelberg F. Antihypertensive treatment, high triglycerides, and low high-density lipoprotein cholesterol and risk of ischemic heart disease mortality: a 16-year followup in the Copenhagen male study. Metab Syndr Relat Disord.
2010;8(3):215-22.

29. Hadaegh F, Khalili D, Ghasemi A, Tohidi M, Sheikholeslami F, Azizi F. Triglyceride/HDL-cholesterol ratio is an independent predictor for coronary heart disease in a population of Iranian men. Nutr Metab Cardiovasc Dis. 2009;19(6):401-8.

30. Jeppesen J, Hein HO, Suadicani P, Gyntelberg F. High triglycerides/low high-density lipoprotein cholesterol, ischemic electrocardiogram changes, and risk of ischemic heart disease. Am Heart J. 2003;145(1):103-8. 